Books

\section{Advances in Geology of the Porphyry Copper Deposits: Southwestern North America}

\section{PAGE 85}

S. R. Titley (Ed.), The University of Arizona Press, Tucson, xiv +560 pp., 1982, $\$ 35.00$

\section{Reviewed by Stephen E. Kesler}

This book summarizes recent advances in information on the porphyry copper deposits of southwestern North America. It is a sequel to an earlier summary volume published by the same group in 1966. The new volume is indispensable for anyone interested in copper deposits and a valuable reference for those working in fields ranging from the petrology of silicic igneous rocks to the tectonic evolution of western North America. The volume is particularly significant because porphyry coppers are by far the most important class of deposits in terms of global copper reserves and, most importantly, because southwestern North America contains an unusually large number and variety of these deposits.

In using this volume it is important to keep in mind that 16 of the 27 chapters were written largely or entirely by geologists from industry whose first responsibility is to exploration and production rather than to research and publication. It is no small task for these geologists to obtain management permission to write papers about their deposits or, in fact, to find time to write. It is an even tougher job to coordinate a volume such as this with contributions from academia, industry, and government, and we can be grateful to editor Spencer Titley for his commitment to this project.

The volume is divided into two parts. The first part contains 13 articles on selected topics in porphyry copper geology including general studies of the grade, tonnage, and economics of worldwide porphyry copper deposits as well as more specific, southwestern North America-oriented papers on the geologic setting of the deposits; their thermal evolution, fracture, and dike patterns; alteration mineralogy, chemistry, character, and evolution; associated skarn alteration and mineralization; leached cappings; and geochemical exploration techniques. This section also includes more specific papers on primary metal dispersion haloes around the Kalamazoo deposit, the geochemistry of sulfur and copper at the Ray deposit, and the petrology of the Ray igenous rocks. Much, though not all, of the information in this section has appeared in print previously, so the value of these papers must lie in their success in drawing new and useful conclusions. Particular success along this line is seen in the chapters on alteration mineralogy and on leached cappings. The second section includes 13 papers on deposits that had not been described previously in any detail or for which significant new data had become available within the last decade. The papers in this section vary widely in quality, but all provide at least a basic geologic framework for the deposits. Particularly commendable in this section are the attempts that have been made to provide information on the relation between copper and molybdenum grades and related geologic features. Comprehensive descriptions such as this are necessary if we are to develop truly realistic genetic models for these deposits.

Considered in its geologic and editorial context, this volume cannot be criticized to any real extent. Its broadest shortcoming, the failure to propose genetic models for the processes and deposits that are discussed, is actually a realistic reflection of the extreme complexity of these deposits and our still limited research progress on them. The lack of a chapter on geophysical exploration methods for these deposits is in part made up for by information in the recently published 75th anniversary volume of the Society of Economic Geologists. Typographic and illustration errors are remarkably scarce.

This volume will no doubt remain a valuable source of data for students of late magmatic mineralizing processes for many years to come and, it is hoped, a starting point for a third, and final (?) summary volume near the end of the next decade.

Stephen E. Kesler is with the Department of Geological Sciences, University of Michigan, Ann Arbor.

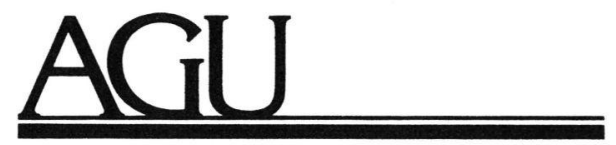

\section{Gelhar Receives Horton Award}

PAGE 86

\section{Citation}

The field of subsurface hydrology was perhaps the last area of hydrology to abandon strict determinism and to incorporate into its analysis the ample uncertainty arising from the structure and properties of the medium. Recent years have seen an explosion of activity in this area, however, made possible in large part by the pioneering contributions of this year's Robert E. Horton Award winner.

His early papers on the spatial variability of aquifer parameters established a general iramework for the treatment of random hydraulic conductivity variations in an aquifer by means of stochastic differential equations. This provided the basis for his important later work on the stochastic theory of macrodispersion in nonuniform porous media. The impact of his contributions is evident from the large number of recent symposia and publications devoted to the stochastic description of groundwater flow and from the fact that his work is referred to extensively in the literature.
He did all his formal academic work at the University of Wisconsin and first joined the MIT faculty in 1964. He left MIT for the New Mexico Institute of Mining and Technology in 1973 and rejoined the MIT faculty this past September. He is a personal friend of long standing and I can think of no one to whom I would rather present the 1982 Robert E. Horton Award than Lynn W. Gelhar.

\section{P. S. Eagleson}

\section{Acceptance}

It is a pleasure to receive this award not only as an indication of individual achievement but also as recognition that stochastic subsurface hydrology has become well established as a legitimate area of research. This has not always been the case. Ten years ago when I first began to work in this area one of the leading groundwater researchers strongly advised me that the stochastic approach was unnecessary because groundwater flow is completely described by deterministic equations. Needless to say I did not heed that advice.

I want to acknowledge the contributions of colleagues at New Mexico Tech and MIT who have worked with me and had an important influence on my thinking in this area. Also, I have been fortunate to have had a succession of sharp graduate students who have worked with me in this area and have benefited from some stimulating competition from individuals like Gedeon Dagan.

I would like to offer some of my perspective on the area of stochastic subsurface hydrology. To me this is more than a question of probabilistic theory or computational methodology. In my view the motivation for a stochastic approach presents itself rather strongly in field observations of the natural variability of properties such as hydraulic conductivity. The key element of the stochastic approach is the coupling of that natural variability with well-established physics which is based on controlled laboratory experiments. The goal is a quantitative description of the controlling, large-scale processes which determine field-scale behavior. The stochastic approach expresses that large-scale behavior in terms of the expected value, and second moment analysis gives a measure of the variability of the prediction about the mean.

The field of stochastic subsurface hydrology has benefited from a diversity of methodologies; comparisons of results have made it possible to develop confidence in the reliability of the various methods. For example, in the very important case of solute transport three distinctly different methods of analysis by independent researchers (Dagan, 1982; Gelhar and Axness, 1983; Winter, 1982) all have produced essentially the same result for the macroscopic dispersion coefficient. The results in this case have important practical implications. First of all, the theory shows that the mean behavior, as reflected in the macrodispersion coefficient, is strongly influenced by the variance of the logarithm of hydraulic conductivity. This is in contrast to the head prediction problem where mean behavior is only weakly dependent on log-conductivity variance. Second, near the source, the mean concentration field is not described by the classical convective dispersion equation; the dispersion coefficients depend oñ displacement distance, and higher order derivatives 